\title{
CHALCHOGENIDE INDUCED INTRAMOLECULAR INTERACTIONS IN [2.2]PARACYCLOPHANES: A REVIEW
}

\author{
LAURA GABRIELA SARBU ${ }^{a}$, LUCIAN GABRIEL BAHRIN ${ }^{a, b}$, \\ HENNING HOPF ${ }^{c}$, MIHAIL-LUCIAN BIRSA ${ }^{\mathrm{a}, *}$
}

\begin{abstract}
The intramolecular interactions in pseudo-geminally substituted [2.2] paracyclophanes induced by chalchogenide halides is reviewed. The subtitution reactions with sulfur dichloride and disulfur dichloride and the addition reactions of selenium dihalides and diselenium dichlorides are highlighted.
\end{abstract}

Keywords: acetylenes, allenes, [2.2]paracyclophane, propargylic alcohols, selenium halides, sulfur halides

\section{INTRODUCTION}

Cyclophanes are strained organic molecules which contain aromatic ring(s) as well as aliphatic unit(s). The aromatic rings provide rigidity to their structure, whereas the aliphatic unit(s) forms bridge(s) between the aromatic rings and also provides flexibility to the overall structure. [2.2]Paracyclophanes ([2.2]PC) are a class of organic compounds which have drawn attention ever since their first appearance in the literature. The molecules of these compounds are made-up of two benzene rings placed one on top of the other, bound together by ethylene bridges in their para positions. The cyclophane chemistry is a fast developing field, as proven by a recent publication by Gleiter and Hopf, which describes the applications of cyclophanes in stereoselective synthesis and the incorporation of cyclophanes in complex molecular structures, like heterocycles and polymers [1]. Initially, [2.2]paracyclophane and its derivatives were studied because of their special geometry, sterical

\footnotetext{
a Alexandru loan Cuza University of lasi, Department of Chemistry, 11 Carol I Blvd., 700506 lasi, Romania

b Petru Poni Institute of Macromolecular Chemistry, Aleea Grigore Ghica Vodă 41A, 700487 lasi, Romania

c Technical University of Braunschweig, Institute of Organic Chemistry, Hagenring 30, D38106 Braunschweig, Germany

*Corresponding author: Ibirsa@uaic.ro
} 
properties, transannular interactions and cycle tension [2]. Because of the rigid molecular frame, recent research suggests using the electronical properties of these compounds in the synthesis of polymers and charge-transfer complexes [3]. A number of cyclophanes have been designed and developed over the years for the selective recognition of various guest biomolecules [4]. Functional groups in pseudo-geminally substituted [2.2]paracyclophanes often undergo highly specific reactions. This is due to the rigid framework and the short distance between the two aromatic rings within the [2.2]paracyclophane unit. Thus, unsaturated cyclophane bis(esters) undergo intramolecular photocyclization, yielding the corresponding ladderane isomers [5-7].

\section{The interaction of pseudo-geminal bis(propargylic)[2.2]PC alcohols with chalcogenide halides}

Using pseudo-geminally substituted [2.2]paracyclophanes as spacers for bis(allenic) moieties, interesting starting materials for intra- or intermolecular reactions can be realized. An accessible entry to allenic systems consists of the $[2,3]$ sigmatropic rearrangement of propargylic sulfenates to allenic sulfoxides. This reaction, which takes place spontaneously at low temperature, has been applied extensively in organic synthesis $[8,9]$. In order to follow this reaction type, the first step was to prepare pseudo-geminal bis(propargylic) alcohols $2 \mathbf{a}-\mathbf{c}$ (Scheme 1) by the reaction of the corresponding lithium acetylide with 4,15-diformyl[2.2]paracyclophane (1) [10]. To avoid side reactions the best results were obtained by adding 1 to the acetylenic salt solution, at $-35^{\circ} \mathrm{C}$ to $-40^{\circ} \mathrm{C}[11]$.

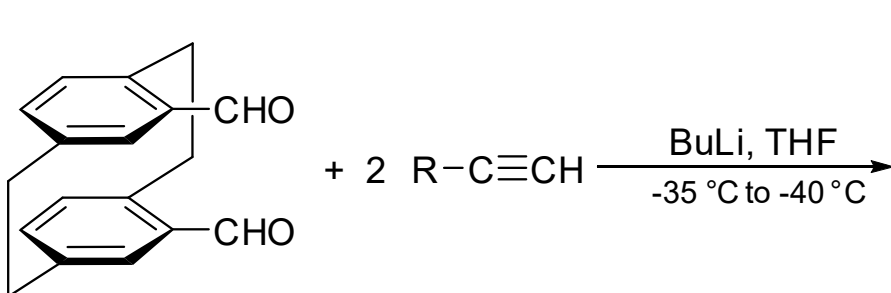

1

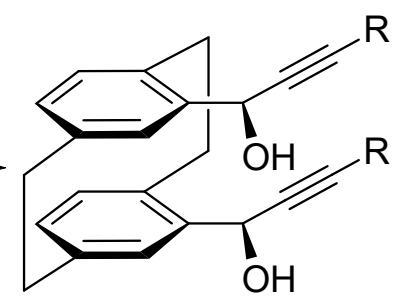

$2 a-c$

2a $\mathrm{R}=n-\mathrm{Pr}, 89 \%$; 2b $\mathrm{R}=n-\mathrm{Bu}, 86 \% ; 2 \mathrm{c} \quad \mathrm{R}=\mathrm{Ph}, 85 \%$

Scheme 1. Synthesis of pseudo-geminal bis(propargylic) alcohols 2a-c

The next step in the synthesis of pseudo-geminal bis(allenic) sulfoxides was to react the bis(propargylic) alcohols of type 2 with the most stable sulfenylchloride, perchloromethylmercaptane (PCMM). As expected, the double [2,3]sigmatropic rearrangement of the initially produced pseudo-geminal bis(propargylic) 
trichloromethylsulfenates takes place spontaneously at low temperature affording the desired pseudo-geminal bis(allenyl) trichloromethylsulfoxides $\mathbf{3 a , b}$ in good yields (Scheme 2) [11]. Both bis(allenyl) sulfoxides have been obtained as a mixture of four inseparable diastereoisomers. By using dimethyldioxirane (DMDO), a particularly mild oxidizing agent for our substrates, we obtained the desired pseudo-geminal bis(allenyl) sulfones $\mathbf{4 a} \mathbf{a}, \mathbf{b}$ as single isomers in moderate yields (Scheme 2).

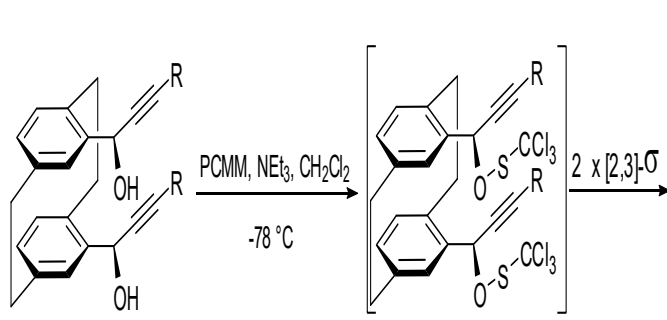

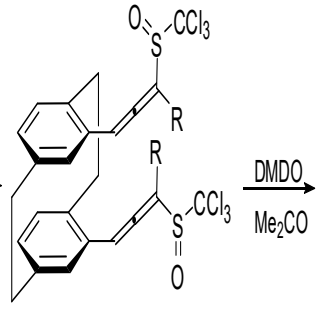

3a $R=n-P r, 84 \%$ $3 b \mathrm{R}=n-B u, 80 \%$

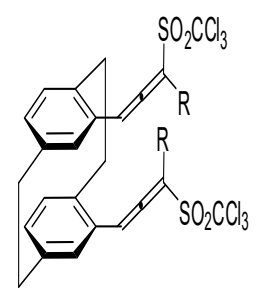

4a $R=n-\operatorname{Pr}, 40 \%$ 4b $R=n-B u, 42 \%$

Scheme 2. Synthesis of pseudo-geminal bis(allenyl) sulfones 4

Although the pseudo-geminally substituted [2.2]paracyclophane core holds the allenic moieties in favorable positions for further intramolecular interactions, none of these have been observed under various conditions. The lack of reactivity of these unsaturated systems could be due to both electronic and steric effects of the trichloromethyl sulfoxide or sulfone substituents, respectively.

Further investigations involved the replacement of the trichloromethyl group with a nitrophenyl substituent [12]. This was accomplished by the reaction of bis(propargylic) alcohols $\mathbf{2 a}$,d with o-nitrobenzenesulfenyl chloride via a double $[2,3]$ sigmatropic rearrangement of the corresponding sulfenyl esters (Scheme 3) $[8,9]$.

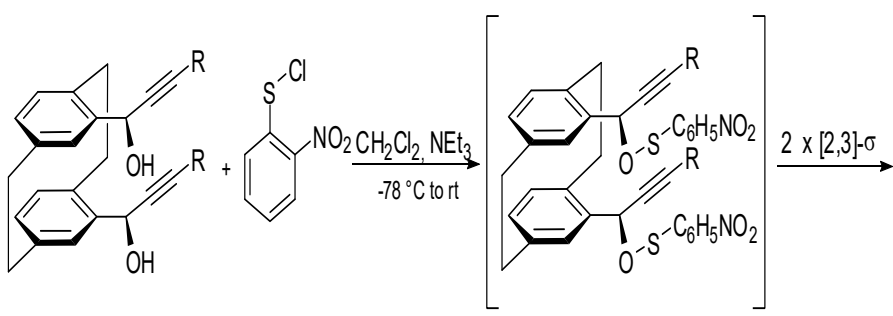

$2 a, d$

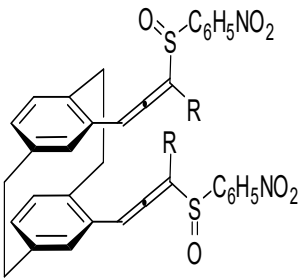

$5 a, b$

$5 \mathrm{a} \mathrm{R}=n-\mathrm{Pr}, \mathrm{C}_{6} \mathrm{H}_{4} \mathrm{NO}_{2}$ (ortho) 5b R $=\mathrm{Me}, \mathrm{C}_{6} \mathrm{H}_{4} \mathrm{NO}_{2}$ (ortho)

2a $\mathrm{R}=n-\mathrm{Pr}$

$2 \mathrm{~d} R=\mathrm{Me}$ 
A solution of 5 was then gently heated at $40^{\circ} \mathrm{C}$ and after purification and separation of the crude mixture, two major compounds were isolated, an $\alpha, \beta$-unsaturated ketone 6 and bis(o-nitrophenyl) disulfide 7 (Scheme 4) [12].

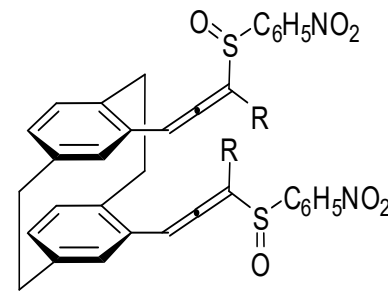

$5 a, b$

5a $\mathrm{R}=n-\mathrm{Pr}, \mathrm{C}_{6} \mathrm{H}_{4} \mathrm{NO}_{2}$ (ortho)

5b $\mathrm{R}=\mathrm{Me}, \mathrm{C}_{6} \mathrm{H}_{4} \mathrm{NO}_{2}$ (ortho)

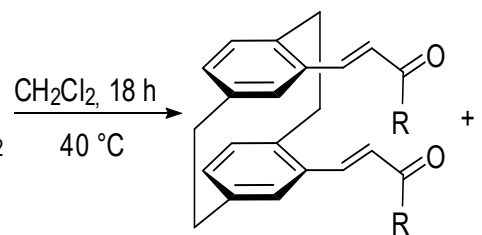

$6 a, b$

6a $\mathrm{R}=n-\mathrm{Pr}$

6b $R=M e$

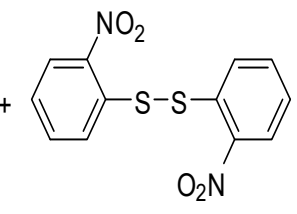

7

Scheme 4. Intramolecular interactions in pseudo-geminal bis(allenyl) nitrophenylsulfoxides

A survey of literature data on ortho-substituted phenyl sulfoxides revealed that mono-ortho-substitution prevents free rotation of the $-S(O) R$ group. Consequently, it is reasonable to assume that the formation of $\alpha, \beta$ unsaturated ketone is initiated by an interaction between the electron pairs of the sulfur and nitrogen atom, respectively, making the system flat and inducing a high rotational barrier around the S-Car bond [13]. Thus, the conversion of $\mathbf{5 a}, \mathbf{b}$ to $\mathbf{6 a , b}$ has involved the formation of a diradical intermediate of type $\mathbf{8}$ which then undergoes a dyotropic rearrangement as presented in Scheme 5 $[14,15]$.

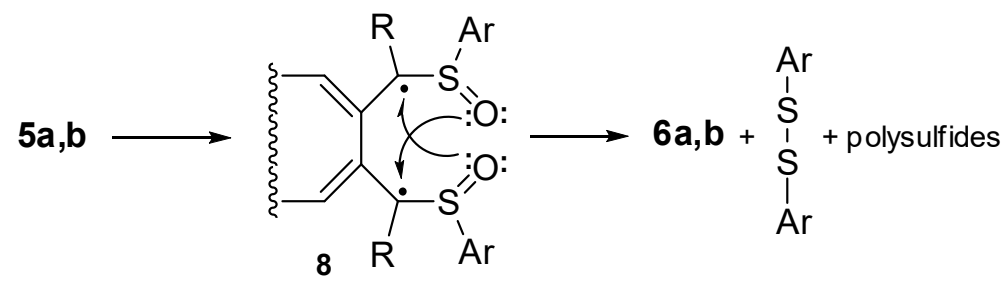

Scheme 5. The mechanism for the synthesis of bis $(\alpha, \beta$-unsaturated) ketones 6

Using the same type of substrate, the reactivity of the disulfur dichloride and sulfur dichloride towards pseudo-geminally substituted propargylic alcohols has been investigated. Thus, the reactions of pseudo-geminal bispropargylic alcohols 2c,d with disulfur dichloride have been performed under high dilution conditions in the presence of triethylamine at $-78{ }^{\circ} \mathrm{C}$ (Scheme 6) [16]. 


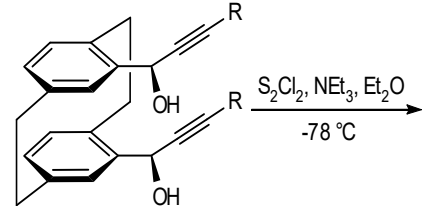

2c, d

$2 c \mathrm{R}=\mathrm{Ph}$ $2 \mathrm{~d} R=\mathrm{Me}$

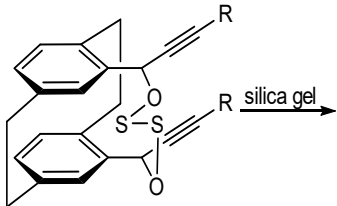

$9 \mathrm{a}, \mathrm{b}$

$9 \mathrm{a} R=\mathrm{Ph}$

$9 b R=M e$

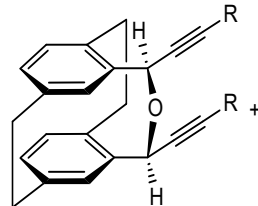

meso-10a,b

$10 \mathrm{a} R=\mathrm{Ph}$

$10 \mathrm{~b} R=\mathrm{Me}$

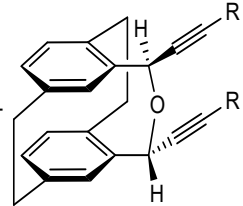

$d l-11 a, b$

$11 \mathrm{a} R=\mathrm{Ph}$

$11 \mathrm{~b} R=\mathrm{Me}$

Scheme 6. Reaction of bis(propargylic) alcohols with disulfur dichloride

Purification of compounds 9 on silica gel, that provides mild acidic conditions, was always accompanied by sulfur extrusion, most likely as polysulfides, and by the formation of cyclic ethers 10 and 11. The formation of cyclic ether from bispropargylic alcohols has also been observed by reacting the latter with pyridinium chloride [17]. The acid catalyzed rearrangement of dipropargyloxy disulfide 9 to the corresponding cyclic ethers appears to be kinetically favored, unlike the double sigmatropic rearrangement to the bisallenyl sulfone derivatives.

Using sulfur dichloride, a mixture of cyclic ethers 10 and 11, and a compound related to disulfide 9 were obtained [16]. The dipropargyloxy sulfides 12 turned out to be more sensitive to acidic conditions provided by silica gel in the work-up process than the corresponding disulfides. This assumption was supported by an unexpected conversion of the crude reaction mixture to a single pinacolone type compound (13), process that involves an intramolecular hydride transfer (Scheme 7) [15].

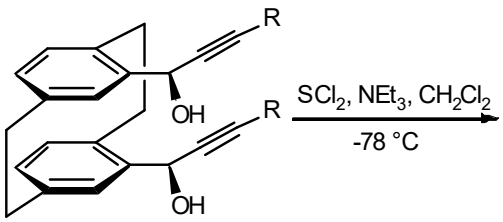

2c,d

2c $\mathrm{R}=\mathrm{Ph}$

$2 \mathrm{~d} R=\mathrm{Me}$

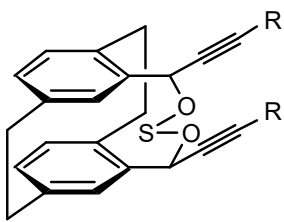

$12 a, b$ $12 \mathrm{a} R=\mathrm{Ph}$
$12 \mathrm{~b} R=\mathrm{Me}$

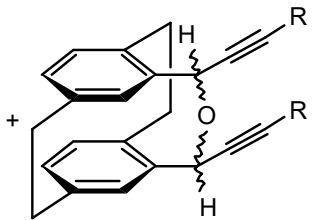

$10 a, b+11 a, b$

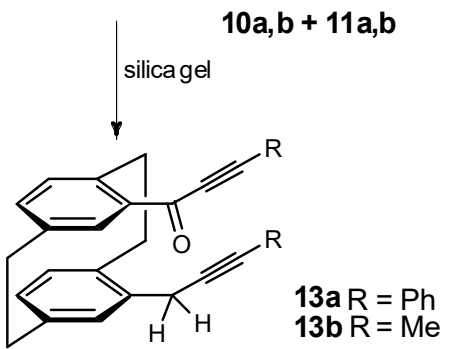

$13 a, b$

Scheme 7. Reaction of bis(propargylic) alcohols with sulfur dichloride 
The regio- and stereospecific addition of monoselenium monochloride to pseudo-geminally substituted bis(propargylic) alcohols has been performed under high dilution conditions. Along with the expected diselenides 14, the monoselenides 15 and triselenides 16 were obtained (Scheme 8) [18]. The disproportionation reaction of selenium monochloride to selenium dichloride and triselenium dichloride [19] leads to the corresponding divinylic mono- and triselenides. For the trans-formation described in Scheme 8, in all cases synaddition with anti-Markovnikov orientation was observed.

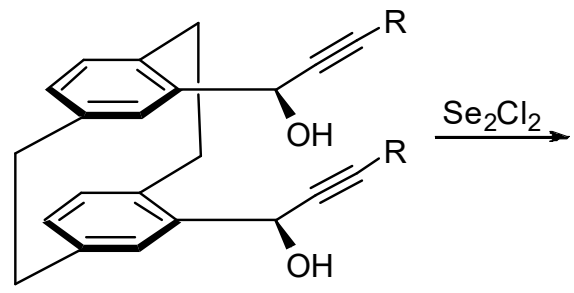

2c, d

2c $\mathrm{R}=\mathrm{Ph}$

2d $R=M e$

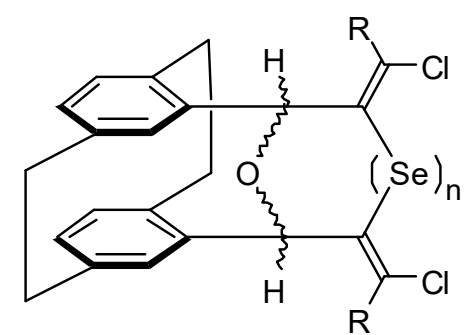

14a $R=P h, 14 b R=M e, n=2$

15a $R=P h, 15 b R=M e, n=1$

16a $R=P h, 16 b R=M e, n=3$

Scheme 8. Addition of $\mathrm{Se}_{2} \mathrm{Cl}_{2}$ to pseudo-geminally substituted bis(propargylic) alcohols

\section{The interaction of pseudo-geminal bis(ethynyl)[2.2]PC with chalcogenide halides}

Following our interest in the introduction of new bridges to [2.2]paracyclophanes, we decided to investigate the reactivity of the chalchogenide halides towards 4,13-bis(ethynyl)[2.2]paracyclophanes. The ethynyl group is well known for its ability to undergo coupling reactions, making the pseudo-geminal bis(acetylene) 17 and its derivatives good candidates for building molecular scaffolding [20,21]. Thus, the reaction of pseudo-geminal bis(acetylene) 17 with $n$-BuLi in THF followed by treatment with $\mathrm{SCl}_{2}$ resulted in the formation of thiophene substituted paracyclophane derivative 19 (Scheme 9) [22]. Most likely the cycloaromatization is induced by a nucleophilic attack of a THF-anion on one of the acetylelenic carbon atoms of the unstable intermediate sulfide 18. 


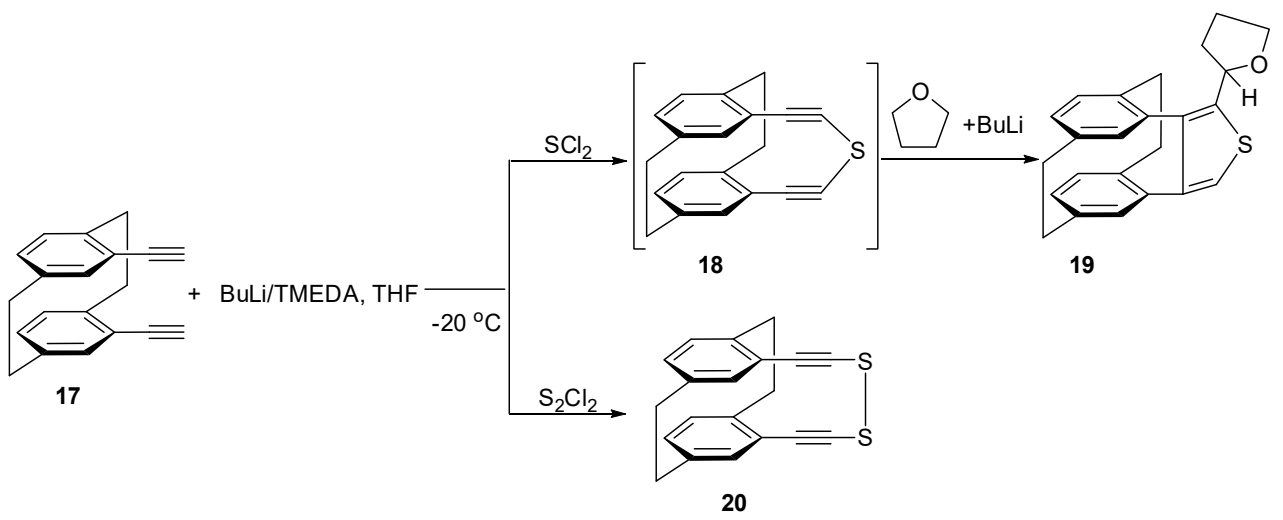

Scheme 9. Addition of $\mathrm{SCl}_{2}$ and $\mathrm{S}_{2} \mathrm{Cl}_{2}$ to 4,13-bis(ethynyl)[2.2]paracyclophane

Under the same reaction conditions, the treatment of bis(acetylene) 17 with monosulfur monochloride led to the corresponding disulfide 20 , in $65 \%$ isolated yield (Scheme 9) [22]. The stability of this compound could be explained by a less hindered structure than that of the monosulfide 18 and the lack of cycloaromatization.

Other investigations involved the interactions of $\mathrm{Se}_{2} \mathrm{Cl}_{2}$ with pseudogeminal bis(acetylene) 17. The addition of electrophilic selenium reagent produced preferably the corresponding E-adduct, diselenide 21, through an anti-addition with Markovnikov orientation (Scheme 10) [22].

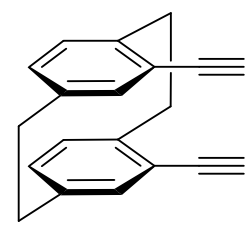

17

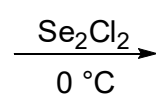

Scheme 10. Addition of diselenium dichloride to 4,13bis(ethynyl)[2.2]paracyclophane

An addition/elimination sequence of selenium halides to pseudogeminally bis(acetylene) substituted [2.2]paracyclophanes leads to new bridges with an endo-exo-diene substructure. Despite of the above reaction outcome, the addition of selenium dichloride and selenium dibromide to pseudo-geminal bis(ethynyl)[2.2]paracyclophane $\mathbf{1 7}$ provided a mixture of [2.3.2] $(1,2,4)$ cyclophane derivatives $\mathbf{2 2}, \mathbf{2 3}, \mathbf{2 5}, \mathbf{2 6}$ and tetrahaloderivatives 24, 27 (Scheme 11) [23]. 


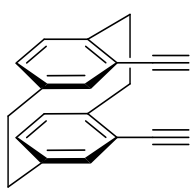

17

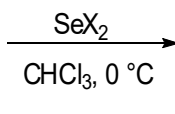

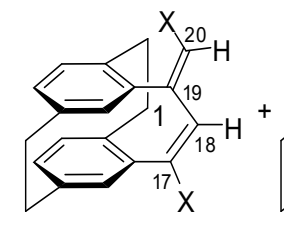

22: $\mathrm{X}=\mathrm{Cl}$

25: $\mathrm{X}=\mathrm{Br}$

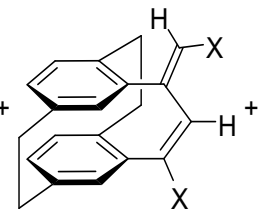

23: $\mathrm{X}=\mathrm{Cl}$

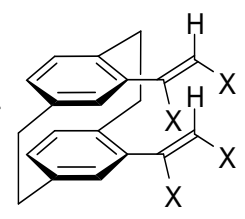

24: $\mathrm{X}=\mathrm{Cl}$ 27: $\mathrm{X}=\mathrm{Br}$

Scheme 11. Reactions of selenium dichloride and selenium dibromide with 4,13-bis(ethynyl)[2.2]paracyclophane

The configuration of compounds 22 and 25 as a (17E,19E)-diene was established from the mutual NOEs between $\mathrm{H} 18$ and $\mathrm{H} 20$. The relative $Z$ stereochemistry of tetrabromo derivative 27 was unambiguously established by X-ray analysis. The unexpected formation of [2.3.2](1,2,4)cyclophane derivatives 22, 23 and 25, 26 prompted the investigation of the addition of phenylselenyl chloride to 4,13-bis(ethynyl)[2.2]paracyclophane. Surprisingly, the addition of 2 eq. of $\mathrm{PhSeCl}$ to bis(acetylene) 17 again provided a mixture of dienes 22 and 23 along with diphenyl diselenide. In a first step, the addition of one equivalent of $\mathrm{PhSeCl}$ to one of the triple bonds of 17 results in the formation of episelenonium ion 28 (Scheme 12). The episelenonium ion 28 should equilibrate with the ringopened form, a benzylic type carbocation; the interaction of this intermediate with the opposing ethynyl substituent provides adduct 29. For steric reasons, the chloride anion attacks from "outside" leading to intermediate 30 . The reaction of 30 with the second equivalent of $\mathrm{PhSeCl}$ leads to selenonium ion 31; once the diphenyl diselenide leaving group is formed, the addition of chloride counter-anion from both directions is accompanied by the formation of [2.3.2] $(1,2,4)$ cyclophane derivatives 22 and 23.

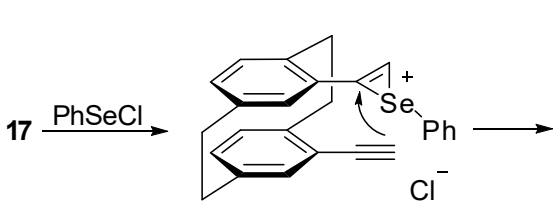

28

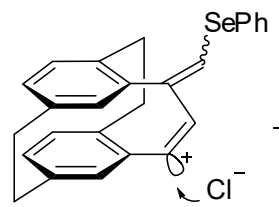

29

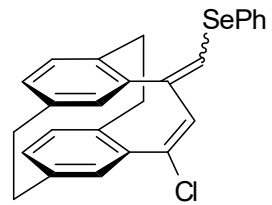

30

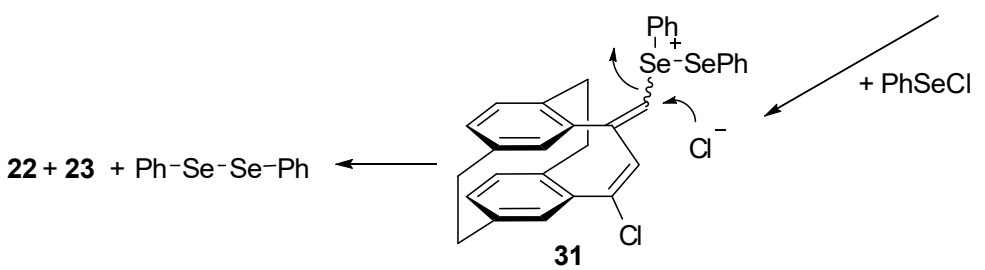

Scheme 12. Addition of phenylselenyl chloride to 4,13-bis(ethynyl)[2.2]paracyclophane 
With regard to the addition of selenium dihalides to bis(acetylene) 17 , the reaction mechanism should follow a similar course, consisting of the formation of a selenonium ion of type $\mathbf{3 0}$ rather than the addition of the selenium electrophiles to the second triple bond. This involves elimination of diselenium dihalides with the formation of dienes 22, 23 and 25, 26.

These reactions have been found to be sensitive to the substitution of the acetylenic bond. Thus, by reacting bis(acetylene) 32 with 1 eq. of selenium dichloride only the $(17 E, 19 E)$-diene 33 and tetrachloride derivative $\mathbf{3 4}$ were isolated (Scheme 13) [23]. The lack of isomeric diene $(17 E, 19 Z)$ could be explained as the result of steric hindrance induced by the presence of methyl groups at the acetylenic carbon atoms. This forces the addition of a chloride anion to a methylated intermediate of type 31 to take place in the way that provides only the thermodynamically stable $(17 E, 19 E)-[2.3 .2](1,2,4)$ cyclophane derivative 33.

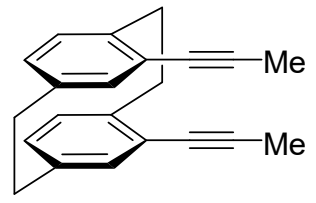

32
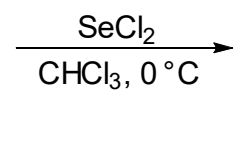

33

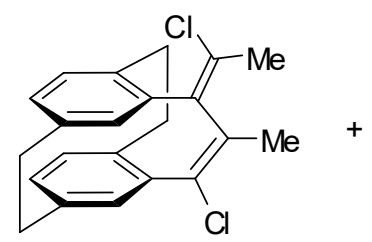

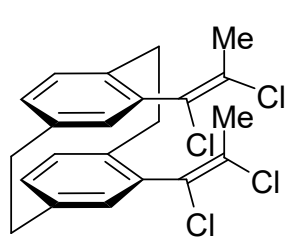

34

Scheme 13. Reactions of selenium dichloride with

4,13-bis(propyn-1-yl)[2.2]paracyclophane 32

\section{CONCLUSIONS}

The reactions of pseudo-geminal bis(ethynyl)[2.2]PC and of pseudogeminal bis(propargylic)[2.2]PC alcohols with chalchogenide halides are presented. The intramolecular interactions generated by these interactions are underlined.

\section{ACKNOWLEDGMENTS}

CNCS - UEFISCDI support within PNCDI III is acknowledged by LGS and LGB for project numbers PN-III-PI-I.1-PD-2016-0962 and PN-III-PI-I.1PD-2016-1117, respectively. 


\section{REFERENCES}

1. H. Hopf; R. Gleiter; Modern Cyclophane Chemistry, Wiley-VCH, Weinheim, 2004.

2. F. Vögtle; Cyclophane Chemistry, Wiley, Chichester, 1993.

3. K.M. El-Shaieb; A F.E. Mourad; H. Hopf; Arkivoc, 2006, 193-200.

4. S. Bartoli; S. Roelens; J. Am. Chem. Soc., 2002, 124, 8307-8315.

5. H. Greiving; H. Hopf; P. G. Jones; P. Bubenitschek; J.-P. Desvergne; H. BouasLaurent; Eur. J. Org. Chem., 2005, 558-566.

6. H. Hopf; H. Greiving; C. Beck; I. Dix; P. G. Jones; J.-P. Desvergne; H. BouasLaurent; Eur. J. Org. Chem., 2005, 567-581.

7. For a review, see: H. Hopf; Angew. Chem., 2003, 115, 2928-2931; Angew. Chem. Int. Ed., 2003, 42, 2822-2825.

8. S. Braverman; The Chemistry of Sulfenic Acids and their Derivatives; S. Patai; Wiley, New York, 1990, 311-359.

9. S. Braverman; The Chemistry of Double-Bonded Functional Groups, Suppl. A2; S. Patai; Wiley, Chichester, 1989, 963-1060.

10. H. Hopf; F.-W. Raulfs; D. Schomburg; Tetrahedron, 1986, 42, 1655-1663.

11. M.L. Birsa; P.G. Jones; S. Braverman; H. Hopf; Synlett, 2005, 4, 640-642.

12. M.L. Birsa; H. Hopf; Synlett, 2007, 17, 2753-2755.

13. V. Baliah; A. Ekambaram; J. Indian Chem. Soc., 1991, 68, 272-278.

14. For reviews see: (a) V. I. Minkin; L. P. Olekhnovich; Yu. A. Zhdanov; Molecular Design of Tautomeric Compounds; D. Reidel Publishing Co.: Dordrecht, 1988, 221-246. (b) M. T. Reetz; Adv. Organomet. Chem., 1977, 16, 33-65.

15. K. Mackenzie; G. Proctor; D.J. Woodnutt; Tetrahedron, 1987, 43, 5981-5993, and references cited therein.

16. A. Birsa; L. Ignat; H. Hopf; M.L. Birsa; Acta Chem. lasi, 2009, 17, 187-196.

17. M.L. Birsa; P.G. Jones; H. Hopf; Eur. J. Org. Chem., 2005, 3263-3270.

18. M.L. Birsa; H. Hopf; Heteroatom Chem., 2010, 21, 126-130.

19. M. Lamoureux; J. Milne; Can. J. Chem., 1989, 67, 1936-1941.

20. L. Bondarenko; I. Dix; H. Hinrich; H. Hopf; Synthesis, 2004, 2751-2759.

21. L. Bondarenko; S. Hentschel; H. Greiving; J. Grunenberg; H. Hopf; I. Dix; P. G. Jones; L. Ernst; Chem. Eur. J., 2007, 13, 3950-3963.

22. L.G. Sarbu; A. Birsa; H. Hopf; M.L. Birsa; Phosphorus, Sulfur and Silicon, 2011, 186, 1246-1250.

23. L.G. Sarbu; H. Hopf; P.G. Jones; M.L. Birsa; Beilstein J. Org. Chem., 2014, 10, 2550-2555. 